\title{
Critique et engagement dans la rhétorique des
} mouvements sociaux

Criticism and Commitment in Social Movement Rhetoric

\section{Eithan Orkibi}

\section{OpenEdition}

1 Journals

Édition électronique

URL : http://journals.openedition.org/aad/1559

DOI : 10.4000/aad.1559

ISSN : $1565-8961$

Éditeur

Université de Tel-Aviv

Référence électronique

Eithan Orkibi, "Critique et engagement dans la rhétorique des mouvements sociaux », Argumentation et Analyse du Discours [En ligne], 11 | 2013, mis en ligne le 15 octobre 2013, consulté le 23 septembre 2019. URL : http://journals.openedition.org/aad/1559; DOI : 10.4000/aad.1559

Ce document a été généré automatiquement le 23 septembre 2019.

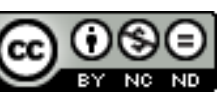

Argumentation \& analyse du discours est mis à disposition selon les termes de la licence Creative Commons Attribution - Pas d'Utilisation Commerciale - Pas de Modification 4.0 International. 


\title{
Critique et engagement dans la rhétorique des mouvements sociaux
}

\author{
Criticism and Commitment in Social Movement Rhetoric
}

\author{
Eithan Orkibi
}

Depuis l'émergence de la "rhétorique des mouvements sociaux» (Social Movement Rhetoric) aux États-Unis dans les années 1960, les spécialistes en cette matière ne cachent pas leur volonté de se positionner par rapport à leur objet de recherche ${ }^{1}$. Qu'il s'agisse de mouvements historiques comme le mouvement abolitionniste ou suffragiste, ou contemporains tels que la protestation afro-américaine ou les hippies, ces chercheurs ont dans un premier temps lutté pour faire reconnaitre le discours des mouvements sociaux comme un objet légitime de critique rhétorique ${ }^{2}$. Leur démarche exploratoire s'est donné pour objectif de convaincre leurs pairs de l'intérêt de la nature rhétorique d'un terrain jusqu'alors négligé: le langage et le travail persuasif accompagnant l'agitation sociale, la mobilisation et l'action collective, dont la parole semble au premier abord très éloignée de ce qu'on peut attendre d'un orateur dit classique. Toute une gamme nouvelle d'orateurs, de styles, de dispositifs et de modes d'expression a ainsi été présentée comme participant pleinement du système global de la fabrication des idées et de la formation des opinions sur la scène publique.

En un deuxième temps, il s'est agi pour les chercheurs de faire face aux enjeux idéologiques de leurs objets de recherche. Dès lors que le discours de la contestation sociale est plus ou moins reconnu dans sa dimension rhétorique, quel doit être le rôle $\mathrm{du}$ chercheur par rapport aux appels et messages issus du mouvement qu'il explore ? L'analyse rhétorique d'un mouvement social contemporain participe-t-elle du processus de diffusion de ses messages ? La critique rhétorique et herméneutique de la parole protestataire ne contribue-t-elle pas à son travail de construction du sens? En choisissant de se pencher tel ou tel mouvement, le chercheur devient-il, bon gré mal gré, son agent dans l'université ? Ces questions, qui n'avaient pas été posées à propos des objets dits classiques de l'analyse rhétorique tels que les grands discours des chefs d'Etat, soulevaient également une série d'interrogations concernant la valeur pédagogique de la rhétorique des mouvements sociaux à l'université. Si, à travers la 
formation rhétorique dans les cours d'initiation et les ateliers de discours public (public speech), les professeurs offrent aux élèves (c'est-à-dire à la future élite professionnelle, économique, juridique et politique de la société) des modèles et des principes propres à la communication efficace, doivent-ils faire de même pour ceux qui souhaitent assumer un rôle actif dans la société civile ? Doivent-ils former aussi des militants, des leaders et des agents sociaux capables de résister à la rhétorique des élites politiques, les initier à formuler des revendications sociales, à agir - et faire agir les autres - en vue d'un changement social?

Ainsi, depuis son émergence, le domaine de la rhétorique des mouvements sociaux est amené à s'interroger plus ou moins ouvertement sur ses propres responsabilités scientifiques et ses fonctions sociales. Le présent article plonge dans ce débat en suivant, en premier lieu, l'émergence et l'évolution de la rhétorique des mouvements sociaux en tant que champ d'études à part entière. Il traite ensuite des questionnements évoqués ci-dessus tel qu'ils sont élaborés et débattus au cours des ans. De cette lecture centrée sur le rôle du chercheur se dégagent deux formes distinctes complémentaires et successives plutôt qu'antagonistes - de ce qu'on peut qualifier d'engagement scientifique et social du chercheur. La première est avancée par des chercheurs qui s'intéressent à la manière dont la critique rhétorique des mouvements sociaux révèle non seulement les traits caractéristiques de la parole protestataire, mais aussi la nature discriminatoire et inégalitaire de l'espace public. Pour eux, l'analyse situationnelle et interactionnelle de la rhétorique des mouvements sociaux exige la redéfinition de certains cadres conceptuels et épistémologiques de la théorie rhétorique, surtout par rapport au jugement éthique de la parole dissidente. La seconde est spécifique aux chercheurs "activistes». Soucieux du rapport entre l'habitus scientifique et la réalité sociale, ils examinent le passage de la théorie à la pratique et contestent les distinctions conventionnelles entre l'université et la rue.

\section{La rhétorique des mouvements sociaux : définition et histoire des enjeux d'une discipline}

La rhétorique des mouvements sociaux devient un domaine de recherche à part entière aux États-Unis dans les années 1960. Les chercheurs en rhétorique et en communication (speech communication) ont répondu de manière favorable à l'appel lancé par Leland Griffin, qui, dans un article daté de 1952 et considéré comme le texte fondateur du domaine, avait appelé à « tourner notre attention de la figure du "grand orateur" singulier» vers "l'étude d'une multiplicité d'orateurs, de discours, d'auditoires et d'occasions » (184)3 . De manière plus spécifique, Griffin suggère dans le même article de commencer par l'analyse rhétorique des mouvements historiques, et y offre un premier modèle d'analyse. Les premiers travaux dans ce sens ont apparu durant les années 1960, et vers la fin des années 1970 l'analyse rhétorique des mouvements sociaux est désormais reconnue comme un domaine d'expertise à part entière, doté de sa propre terminologie, et présentant des tendances diverses ${ }^{4}$. Cette " première génération » des années 1960-70 a également vu émerger en son sein deux approches principales distinctes, dont les différences continuent à structurer l'évolution du domaine : l'approche fonctionnelle et l'approche herméneutique.

L'approche fonctionnelle a pour fondement l'hypothèse selon laquelle « la rhétorique est l'agent (agency) principal par le biais duquel les mouvements sociaux accomplissent 
différentes fonctions qui leur permettent d'exister, de réagir à l'opposition et, peut-être, de réussir à promouvoir (ou à résister à) un changement " (Stewart 1980: 299). Cette approche considère le mouvement social comme un acteur social rationnel, à savoir une collectivité plus ou moins organisée, dont les dirigeants et porte-parole utilisent la rhétorique de façon stratégique afin de réaliser des objectifs préalablement définis. Pour l'approche fonctionnelle, la rhétorique des mouvements sociaux est un instrument mobilisé dans une situation donnée, et le discours doit être étudié dans et en fonction de son contexte. Cette approche explore ainsi les fonctions qu'accomplit la communication persuasive dans les contextes les plus précis et les plus immédiats de l'action collective : la mise en place des revendications, la mobilisation de militants, la gestion de la lutte sociale, la confrontation ou la négociation avec les institutions et les rapports avec l'opinion publique. Les chercheurs y examinent, par exemple, les caractéristiques et les fonctions de la rhétorique en fonction des étapes successives de l'évolution de l'action collective (Bowers et Ochs 1971), ou les modalités à travers lesquelles la rhétorique permet aux dirigeants sociaux d'affronter différents auditoires et de résoudre les dilemmes qui surgissent dans le feu de l'action (Simons 1971). Également étudiée dans cette approche est la manière dont la rhétorique renforce l'esprit militant des membres des mouvements et consolide leur identité (Gregg 1971), et la façon dont les différentes formes linguistiques et stylistiques typiquement associées à la parole protestataire, comme par exemple les slogans (Denton 1980) ou l'usage de mots obscènes (Rothwell 1971), permettent de véhiculer des messages et de constituer des espaces pour la mise en place d'arguments.

L'approche herméneutique s'intéresse beaucoup moins à l'étude de la rhétorique des mouvements sociaux comme type de "discours organisationnel» dont on évalue l'efficacité, les objectifs et les fonctions immédiates. Les tenants de cette approche considèrent qu'en mettant trop l'accent sur l'instrumentalité du discours des mouvements, les «fonctionnalistes » ignorent les différences entre la rhétorique des mouvements sociaux et les stratégies persuasives utilisées par d'autres types de " collectivités organisées ", telles que les entreprises et les grandes compagnies (DeLuca 1999a : 29). Cherchant à saisir de manière plus exacte la spécificité et la singularité de la rhétorique des mouvements sociaux, l'approche herméneutique les considère comme des producteurs de sens. Elle explore les modalités selon lesquelles leur rhétorique aboutit à dramatisation des conflits sociaux (Griffin 1964) ou à la contestation du discours hégémonique (Kampbell 1973). Également fréquent dans cette approche est l'examen du rôle de la rhétorique dans la formation des identités collectives (Lake 1983) et dans la modification de la « conscience sociale » et de la «perception collective de la réalité " (DeLuca 1999a). Les chercheurs dont les travaux relevant de la perspective herméneutique utilisent des modèles théoriques comme l'«idéographe " (ideograph) pour tracer l'évolution de notions et l'usage de mots-clés tels que "liberté » ou " égalité » dans le discours idéologique et politique (McGee 1980); ils se focalisent sur les rapports dialectiques entre le discours protestataire et le discours hégémonique (Cathcart 1978) ou adoptent une approche socio-historique qui conçoit le mouvement social comme une forme de " comportement rhétorique » (Zarefsky 1980). Ainsi, au lieu de traiter le mouvement comme un "phénomène empirique ", c'est-à-dire une entité sociale définie par ses caractéristiques contextuelles (type, dimensions, lieu et temps de l'action), les chercheurs affiliés à l'approche herméneutique y voient plutôt un "ensemble designifications " (set of meanings - McGee 1980), un espace discursif à l'origine de la création et de la diffusion des idées et des notions sur la longue durée. 
Si les chercheurs des deux courants ont renoncé à la tentative d'élaborer une théorie globale et unifiée de la rhétorique des mouvements sociaux ${ }^{5}$, les générations qui ont pris le relais à partir des années 1980 n'ont pas pour autant perdu tout intérêt pour la parole protestataire. Depuis les années 1990, la recherche en rhétorique des mouvements sociaux porte sur des dimensions et aspects extrêmement variés, comme l'évolution des arguments et contre-arguments dans une polémique sociale (Railsback 1984); la musique populaire dans la rhétorique protestataire (Knupp 1981); la rhétorique du corps dans l'action protestataire (DeLuca 1999b); la rhétorique vernaculaire des militants de base (Hauser et mcclellan 2009); ou l'émergence du mouvement comme un hyperénonciateur qui constitue une "agentivité collective" (collective agency - Enck-Wanzer 2006).

La réédition de quelques ouvrage classiques comme The Rhetoric of Agitation and Control (1e édition en 1971, 3e édition en 2010), de livres de synthèse comme Persuasion and Social Movements (Stewart et al., 1e édition en 1984, 6e édition en 2012), la parution d'anthologies de travaux séminaux représentatifs comme Reading on the Rhetoric of Social Protest (Morris et Browne éds, 2001, $2^{\mathrm{e}}$ édition en 2006), et la publication récente de nouveaux ouvrages (Foust 2010, McKenzie Stevens et Malesh 2009, Cloud 2011a) - tout cela témoigne du fait que le discours des mouvements sociaux demeure un objet central pour l'analyse rhétorique (Jensen 2006).

Et, comme nous l'avons noté plus haut, le métadiscours autour de la responsabilité du chercheur et la fonction sociale de la recherche est l'une des caractéristiques les plus marquées des travaux sur la rhétorique des mouvements sociaux. Ceci s'explique avant tout par le fait que ce domaine traite de conflits sociaux et examine l'usage du langage et du comportement symbolique en relation avec les grandes questions qui divisent la société. Aux États-Unis, principalement dans les années 1960-70, cette question de la responsabilité $\mathrm{du}$ chercheur était indissociable d'une réalité sociale vécue par les chercheurs eux-mêmes, en raison de l'engagement du milieu universitaire dans les luttes sociales de la période ${ }^{6}$. Sans aucun doute, les chercheurs en rhétorique des mouvements sociaux s'occupent de ce que Marianne Doury dénomme des «sujets brûlants " (2004) : des mouvements contemporains ou historiques dont les enjeux ont une forte dimension collective face à laquelle le chercheur se positionne avant tout en tant que citoyen et membre d'une élite intellectuelle : idéologies, conflits de pouvoir, identités, religions, droits humains, etc.

Mais au-delà des questions brûlantes, le métadiscours autour de la responsabilité du chercheur tient aussi aux conditions d'émergence et d'évolution du domaine. La première génération de chercheurs a fait ses premiers pas sous l'influence de la sociologie des mouvements sociaux. Les années 1960-70, période si riche en mobilisations, marquent non seulement l'émergence de ce domaine, mais aussi les transformations qui se font jour dans la sociologie de l'action collective. Celle-là connaît à cette époque une évolution des conceptualisations théoriques, et voit surtout fleurir les travaux affiliés au courant de la «mobilisation des ressources ». Ce dernier, en rupture avec la théorie du « comportement collectif» qui explique le mouvement social en termes de "déviance" ou de "politique de l'impatience " (Chazel 2009), souhaite reconnaitre le "choix rationnel » qui s'effectue dans l'action collective en mettant l'accent sur les aspects organisationnels et professionnels du processus de mobilisation, et sur la logique de celle-ci comme moyen de participation politique. Dans la mesure où cette démarche constitue elle-même une prise de position du chercheur 
par rapport à son objet de recherche, il n'est pas étonnant de constater que les chercheurs en rhétorique empruntent aux sociologues non seulement quelques hypothèses et définitions clés, mais aussi la nécessité de s'interroger sur leurs propres présupposés vis-à-vis de leurs objets.

Ensuite, et plus étroitement en lien avec la théorie rhétorique : la communication des mouvements sociaux n'est pas toujours verbale et textuelle, ni forcément structurée autour de "bons arguments" formulés sur le modèle des manuels scolaires en « composition ». En fait, les mouvements s'expriment souvent à travers des menaces et ultimatums, des slogans scandés et apposés sur les murs, et des actions symboliques comme l'occupation de locaux ou l'outrage au drapeau. En conséquence, tout un débat se poursuit autour de la nature "rhétorique» de la communication des mouvements sociaux : comment doit-on définir la rhétorique au préalable, et quelle idéologie soustend chacune des définitions données au terme de rhétorique? Ce type de problématiques amène les chercheurs à se confronter à des dilemmes qui renvoient à la validité scientifique et aux implications morales de leurs propres hypothèses théoriques.

Ces enjeux constituent ainsi l'arrière-plan du débat auquel nous allons consacrer la suite de cet article, tout en réinvestissant des remarques et des affirmations exprimées par les chercheurs au cours des années à la fois par rapport à la théorie rhétorique et sa pertinence pour le discours contestataire, et par rapport au rôle que jouent la recherche et l'enseignement de la rhétorique des mouvements sociaux à l'égard de la réalité sociale.

\section{L'ouverture du ghetto rhétorique par la première génération}

Les premiers travaux en rhétorique des mouvements sociaux ont été réalisés dans un contexte de suspicion, voire d'hostilité, par rapport au langage et aux tactiques utilisés par les militants des mouvements 7 . Dans un article publié en 1967, Franklyn S. Haiman décrit deux types de critique que les milieux enseignant, institutionnel et politique ont énoncés contre la langue et le comportement des mouvements contemporains. Le premier porte sur la nature illégale d'une partie des tactiques mises en œuvre par les mouvements comme la perturbation de la circulation par le blocage de la voie publique, l'occupation des locaux (sit-in) ou l'appel à la désobéissance. L'autre ligne de critique cible plus concrètement la nature de la « rhétorique de la rue » qui recourt aux appels à l'émotion, voire à la force :

La nouvelle rhétorique dépasse les limites du discours rationnel que les enseignant de rhétorique valorisent si fortement et à la promotion duquel ils se consacrent ; la nouvelle rhétorique est "persuasion » par une stratégie de force et de coercition plutôt que par la raison et par des modalités démocratiques de prise de décisions (102).

Haiman ne prétend pas réfuter cette critique portée contre la « rhétorique de la rue ». Il attire néanmoins l'attention sur les contraintes situationnelles souvent imposées aux militants et dirigeants des mouvements, et note qu' «il n'est pas facile de préciser la mesure dans laquelle le processus démocratique et donc la possibilité d'user d'un discours soumis aux lois de la raison sont effectivement à la portée » de tous. Voici le message qu'il adresse à ses collègues, chercheurs et enseignants en rhétorique : 
Peut-être le mieux qu'on puisse faire est-il d'éviter la présomption optimiste que les canaux de communication rationnelle sont ouverts à tous ceux qui souhaitent les utiliser et tenter plutôt une évaluation minutieuse de la structure de pouvoir inhérente à la situation. Dans la mesure où on découvrirait un déséquilibre du pouvoir et une réticence concomitante de la part des institutions à s'engager dans un véritable dialogue, on serait sans doute moins sévère dans la critique de ceux qui cherchent à rétablir l'équilibre à l'aide de stratégies de persuasion non rationnelles (114).

N'abandonnant pas la division traditionnelle qui sous-tend la critique rhétorique, celle qui distingue entre "émotion" (inférieure) et "rationalité » (supérieure), et pour laquelle la «raison » est le critère principal de l'évaluation, Haiman suggère d'ajouter une dimension supplémentaire : celle qui s'interroge sur les conditions et les objectifs en fonction desquels l'orateur choisit, délibérément, de passer à la " persuasion non rationnelle». Cette démarche implique, pour le chercheur, de "rationaliser» l'« irrationnel », c'est-à-dire de distinguer entre l'orateur et le message : un message non-rationnel peut désormais être le produit d'un choix stratégique entièrement rationnel fait par un orateur lui-même parfaitement rationnel. En conséquence, le jugement éthique de la persuasion non-rationnelle - de l'appel aux émotions, par exemple - n'est plus basé sur le message rhétorique lui-même, mais sur une lecture critique de la situation où il est produit. La question de savoir si tel ou tel message viole les impératifs moraux est moins liée au message lui-même, qu'aux conditions qui guident le choix de l'orateur. Dans la proposition de Haiman, on trouve donc l'hypothèse implicite selon laquelle dans certains contextes, l'immoralité réside non pas dans la "persuasion non rationnelle », mais plutôt dans la structure de la situation.

Un appel similaire aux rhétoriciens leur demandant de réexaminer leurs hypothèses de travail est lancé dans un article rédigé par Robert Scott et Donald Smith en 1969, postulant que la confrontation, dans ses diverses manifestations, est principalement symbolique. Tandis que Haiman souligne la spécificité de la situation rhétorique au sein de laquelle les mouvements agissent, Scott et Smith identifient la dimension symbolique des différentes stratégies de protestation. Il s'agit d'une démarche importante, qui ne cherche pas à « justifier » une " persuasion non-rationnelle » par la situation de l'orateur dans une structure sociale donnée, mais plutôt à concevoir les modes d'expression typiques de la confrontation comme une langue symbolique à part entière ${ }^{8}$. Dans leur conclusion, les auteurs posent que

Depuis Aristote, la rhétorique académique s'est faite principalement l'instrument de la société institutionnalisée, présupposant les «bienfaits " de l'ordre, de la civilité, de la raison, du décorum, du droit civil ou théocratique. Même si les présupposés de civilité et de raisonnement sous-jacents à la vieille rhétorique restent valides, ils ne peuvent pas être considérés comme évidents. Une théorie rhétorique adaptée à notre époque doit tenir compte de l'accusation selon laquelle la civilité et le décorum peuvent servir de masque pour la préservation de l'injustice. [...] Nous qui professons la rhétorique, nous devons déchiffrer la rhétorique de confrontation et chercher à comprendre ses présupposés, ses tactiques et ses objectifs (7-8).

Au-delà du souci de trouver, dans la théorie rhétorique, les outils permettant de mieux rendre compte des modalités de la rhétorique des mouvements sociaux, les appels lancés à cette époque ciblent ouvertement la nature normative de la discipline telle que professée dans les universités. Or, il ne s'agit pas d'abandonner le régime normatif et de promouvoir une approche purement descriptive, mais plutôt de mettre à jour les valeurs constitutives de la théorie et de reconstruire son éthique en posant un regard 
plus critique sur la réalité sociale. Les chercheurs sont ainsi appelés à reconsidérer leur conception d'une "bonne" rhétorique, dont le bon fonctionnement repose sur une conception erronée de la structure des opportunités - c'est-à-dire des chances offertes aux uns et aux autres dans la société . A l'encontre de l'idée qui pose que des " arguments rationnels » donnent à tous l'occasion d'exprimer leur voix sur la scène publique dans un système pluraliste de prise de décisions, les rhétoriciens sont appelés à admettre que l'espace public est préalablement réservé à certains groupes, à savoir à l'élite politique. Un des objectifs de la rhétorique - et de sa critique - serait donc de démasquer ce système et de promouvoir l'accès des outsiders à la scène publique. Ainsi, les auteurs placent au sein de leur interrogation la question de savoir quelle valeur symbolique possède la rhétorique de la confrontation, et quels symboles y sont créés et mobilisés pour modifier la situation rhétorique.

Une tentative d'ainsi « lire la rhétorique de confrontation » est réalisée par Parke G. Burgess dans une série d'articles consacrés à la rhétorique du «Black Power Movement ». A l'instar de Haiman et de Scott et Smith, Burgess envisage la rhétorique de confrontation dans d'une situation d'injustice. La parole dissidente, celle qui choque le public, a pour objectif d'exposer les tensions sociales et de forcer la société à faire face aux dilemmes moraux. Ainsi, Burgess pose que « derrière tout le bruit et la fureur de cette rhétorique réside tout simplement l'intention [de la part des mouvements] d'imposer à la culture une décision morale " par rapport à ces mêmes dilemmes (1968: 123). Dans son article sur la "rhétorique coercitive», il dénonce la division traditionnelle établie entre " persuasion » et « coercition », rejette l'idée que les formes « coercitives » du langage, comme l'ordre ou la menace, appartiennent à une catégorie distincte - celle du "mode impératif du discours» qui n'aurait aucune "force persuasive $\aleph^{10}$, et suggère que la rhétorique coercitive construit, utilise et reconstruit des symboles (1973: 70).

Au-delà d'une communication rhétorique évaluée en fonction de sa capacité d'aboutir à un consensus à travers la mise en place d'arguments rationnels, il est donc impératif d'examiner sa capacité à résoudre l'inégalité de la situation rhétorique à travers, par exemple, la mise en œuvre d'une "rhétorique coercitive" (coercive rhetoric) par des groupes exclus et marginalisés. Plaidant pour une approche pluraliste qui admet l'existence de différentes cultures rhétoriques ${ }^{11}$, Burgess se propose de faire progresser de quelques pas l'appel de Scott et Smith en faveur d'une nouvelle théorie : il suggère au chercheur en rhétorique des mouvements sociaux d'explorer les rapports entre l'aspect stratégique et la dimension morale de chaque action rhétorique. Dans ce qu'il dénomme "l'approche métamorale ", le choix moral de l'orateur fait partie intégrante de son choix stratégique :

La bataille historique entre les moralistes et les amoralistes est inutile de même que la dichotomie qui l'a fait émerger ${ }^{12}$. Équipés d'une perspective métamorale, les critiques peuvent respecter la dimension morale et pourtant rendre à la stratégie ce qui est à la stratégie. Ce faisant, ils remplissent le vide critique dont souffre l'action symbolique lorsqu'on adopte une approche amorale de la stratégie rhétorique. Les effets contrastés sur la critique méritent une analyse, car ce vide englobe aussi la signification de l'action symbolique qui est affiliée à un conflit moral (1970 : 127).

Burgess et ses homologues ont vécu une réalité sociale et observé des phénomènes linguistiques face auxquels, selon eux, les cadres conceptuels de l'analyse n'étaient plus adéquats. Cette nouvelle réalité ( la révolution de notre temps» dans les termes de Burgess) exige, à leurs yeux, une reconceptualisation des cadres théoriques, des 
instruments de recherche et des méthodologies. Les chercheurs de la première génération ont introduit dans le domaine de la critique rhétorique un ensemble de groupes et d'espaces discursifs qui en avaient été jusque-là exclus, et ont modifié en profondeur les paradigmes de la recherche dans leur champ d'investigation. Ils ont ainsi renouvelé la réflexion sur les présupposés sous-jacents aux jugements portés sur les qualités morales et rationnelles du discours.

\section{Les « activistes-académiques » de la nouvelle génération}

Mais la motivation "scientifique" n'est pas forcement la seule. Comme il est explicitement noté par Scott et Smith, "la rhétorique contemporaine de la confrontation est défendue par des théoriciens dont les aspirations à un monde meilleur ne sont pas aisément rejetées, et dont la passion pour l'action est égale ou supérieure à leur passion pour la théorie » (1969: 7). Un article publié par Malcolm 0. Sillars en 1980 peut nous fournir un bon point de départ pour cette approche :

Les mouvements constituent donc des actions collectives perçues par un critique. Elles sont définies par ce critique à travers les événements, conflit et stratégies rhétoriques les plus pertinents, qui peuvent expliquer de la meilleure façon la perspective du critique sur le mouvement. Le critique peut accepter les limites du mouvement telles que fournies par autrui, ou « créer » le mouvement (31, souligné par M. S.).

Le critique - c'est-à-dire le chercheur - est équipé des meilleurs instruments et est donc autorisé à définir le mouvement, à analyser sa signification et interpréter son existence. Autrement dit, il peut «créer » le mouvement en le définissant en tant que mouvement, puis en lui attribuant une signification rhétorique.

Ce passage est cité dans l'introduction d'un ouvrage collectif publié en 2009 sous le titre Active Voices: Composing a Rhetoric for Social Movements. Les coordinatrices de cette publication, Patricia Malesh et Sharon McKenzie Stevens, constatent le «rôle générateur » (generative role) du chercheur qui, en plus et par le fait d'analyser un mouvement, participe à sa création et à son évolution (12). Elles affirment l'importance de «l'étude de la rhétorique d'un mouvement comme une heuristique qui permet au mouvement de se comprendre lui-même », et ajoutent :

En déterminant les caractéristiques importantes qui définissent un mouvement ses objectifs, ses adhérents, sa portée, et ses modalités de persuasion - les chercheurs sont mieux équipés pour comprendre les rapports entre ces caractéristiques et, en fin de compte, la nature des mouvements eux-mêmes. Ils sont également imprégnés de l'esprit du militantisme. En tant qu'activité qui forme le mouvement du point de vue rhétorique, de manière interactive et matérielle, l'acte scientifique de définir un mouvement le fait avancer, transformant ceux qui le définissent en participants actifs dans ce processus (13).

Volontairement ou pas, les chercheurs qui adoptent une approche herméneutique deviennent des participants actifs dans le mouvement social. Cette constatation confère au chercheur une responsabilité scientifique et sociale, non seulement face au projet scientifique (côté théorique), mais aussi face au mouvement qu'il analyse (côté social). En fait, en soumettant un mouvement donné à l'analyse, le chercheur le considère d'abord comme un phénomène suffisamment important ou central pour mériter son attention; ensuite, il le définit et interprète sa signification et par là même permet au mouvement lui-même de s'interroger sur ses principes; et enfin, dans la mesure où il 
est « équipé » et "autorisé » à " expliquer » le mouvement, il prend un rôle actif par rapport à l'action à l'extérieur et à l'intérieur du mouvement. Impossible donc, selon cette approche, d'analyser un mouvement social sans agir en retour sur lui.

Cette vision, dont les origines remontent à Sillars (1980) et McGee (1980), devient particulièrement pertinente - ou pour le moins séduisante - dans le contexte du débat sur l'université et le milieu académique aux États-Unis, surtout depuis le 11 septembre, et face à ce qu'un certain nombre de théoriciens qualifient de « la menace la plus grave sur la liberté académique depuis le maccarthysme des années 1950 » (Doumani 2006 : 11). Pour les coordinatrices de Active Voices, cette période - où " la liberté individuelle et les conflits domestiques s'évanouissent » (2) et où le "capitalisme avancé » fait que « les agences du gouvernement et des corporations continuent à miner les domaines de l'identité individuelle » (4) - exige que « les universitaires-militants (activist academics) reconsidèrent la manière dont on théorise et participe au changement social » (5).

La première démarche impérative est d'éviter la présomption (optimiste) qu'il existe une objectivité dans la production et la diffusion du savoir à l'université, et d'exposer publiquement sa « subjectivité »:

Nous affirmons que le savoir, et les modalités au gré desquelles il est acquis, est toujours le résultat d'intérêts, et à ce titre il est rhétorique. Nous soutenons ensuite que ceux qui participent à la construction du savoir en cachant leur propre rhétoricité, et ceux qui les défendent, sont souvent émotionnellement et matériellement investis dans le statu quo, avec ses inégalités structurelles et partisanes. En explicitant notre aspiration à une théorie et une praxis liées au monde dans lequel nous vivons, et significatives à son égard, nous ne devenons pas plus politiques ou plus idéologiques. Tout simplement, nous exposons notre subjectivité afin de rendre compte des conséquences de notre activité universitaire, et pour encourager une discussion plus large sur la nature transformatrice de l'apprentissage (14).

Le terme d'« universitaires-militants » renvoie ainsi en premier lieu aux universitaires ouvertement engagés dans l'effort d'exposer les rapports entre la production et la diffusion du savoir dans le champ académique et le système d'intérêts politiques, économiques (et militaires, parfois) qui l'anime. Que ce soit la préservation du statu quo ou la transformation sociale, le chercheur est appelé en premier lieu à se confronter aux enjeux politiques de son travail, en rendant compte de «qui» et « quoi » tirent profit du savoir dont il est producteur et diffuseur.

Mais les chercheurs en rhétorique des mouvements sociaux ont une responsabilité supplémentaire qui résulte de leur spécialité (rhétorique) et leur objet d'analyse (le mouvement social). En tant que rhétoriciens qui étudient «l'art et la pratique de la persuasion et de la communication ", les universitaires-militants s'engagent à exploiter la posture unique de la rhétorique - représentée dans plusieurs disciplines en sciences sociales et humaines - pour contester le régime du savoir à l'université, et plus précisément les distinctions disciplinaires ${ }^{13}$. Autrement dit, ce n'est pas uniquement le produit de la recherche qui facilite la préservation du statu quo (ou qui véhiculerait une transformation sociale), c'est aussi le régime du savoir qui doit être reconsidéré :

Ceci implique d'abandonner la revendication disciplinaire $\mathrm{du}$ savoir (l'appropriation du savoir par chaque discipline) et déconstruire le dialogue jargonnant qui accompagne cette revendication. Il s'agit de créer une optique analytique qui soit plus inclusive qu'exclusive, qui soit à la recherche des connexions et non pas des ruptures (5). 
Et enfin, en tant que spécialistes des mouvements sociaux, les universitaires-militants doivent reconnaitre et utiliser la classe - dans le sens littéraire et métaphorique du mot - comme « lieu de possibilités et de résistance »:

En tant qu'universitaires-militants, notre responsabilité consiste à exposer nos étudiants à leur propre agentivité potentielle et à les encourager à l'asserter en les aidant à apprendre comment utiliser les instruments à la croisée de l'analyse, de la critique et de l'action. [...] La classe devient un lieu où l'apprentissage est un moyen non seulement d'acquisition d'un pouvoir, mais aussi d'émancipation et de libération. [...] Dans ce sens, la pratique dans la classe préfigure l'agentivité sociale, citoyenne et personnelle, qui à son tour facilite - crée un espace pour - un changement social. [...] En tant que chercheurs, notre mission est de travailler avec d'autres, que ce soient nos collègues, étudiants, membres de la communauté, juristes, etc., en créant un espace - matériel et discursif - qui facilitera un dialogue et une action transformateurs (16).

Cette aspiration à articuler ou unifier la pratique scientifique et la pratique sociale semble être devenue une raison d'être pour un certain nombre de chercheurs en rhétorique aujourd'hui. Il est vrai, comme en témoignent les essais réunis dans Activism and Rhetoric: Theories and Contexts for Political Engagement (2011), que la tendance à l'engagement n'est pas typique ou spécifique à la rhétorique des mouvements sociaux. Mais les chercheurs en question ne se considèrent pas simplement comme des militants : ils ont pour vocation d'élaborer un discours qui, en analysant et explorant les mots, contribue à faire advenir le changement souhaité.

Cet « esprit de militantisme » et cette « passion pour la théorie et l'action » sont loin de rester implicites dans la conclusion du premier article de ce volume dont l'auteur, Dana L. Cloud, est célèbre pour ses travaux sur la rhétorique de l'action collective :

Je vous encourage à rejoindre et construire des mouvements sociaux, à reconnaitre la valeur des organisations pour le changement social de longue durée, à pousser les autres à s'engager, et à créer et utiliser des espaces pour l'éducation, la délibération et l'action dans la communauté autour de vous. Si vous êtes critiques de notre système, des idéologies qui soutiennent ses horreurs - mettre les idées en action est

la seule chose concevable à faire (Cloud 2011b : 22).

Les « universitaires-militants» expriment ouvertement leur volonté de briser les distinctions établies entre les disciplines scientifiques, entre les deux pratiques universitaires (chercher et enseigner), entre la pratique théorique et la pratique sociale, et enfin, entre la tour d'ivoire de l'académie et la rue. Ils appellent à la transformation de la classe en un lieu d'action et de résistance, et souhaitent ainsi amener - et rejoindre - leurs étudiants dans la rue.

\section{En guise de conclusion}

La longue route parcourue par la rhétorique des mouvements sociaux permet de constater l'émergence de deux formes successives de responsabilité. La première génération, de pair avec les nouveaux courants de la sociologie des mouvements sociaux, a insisté sur le caractère moral et rationnel d'un discours qui, au premier abord, semblait être en contradiction avec l'idéal traditionnel de la théorie rhétorique. Comme leurs homologues en sociologie, les chercheurs en rhétorique ont rejeté l'idée selon laquelle les mouvements de protestation témoignent d'une crise sociale où les masses se livrent à des explosions de violence injustifiées. Ils ont préféré examiner les modalités selon lesquelles les mouvements utilisent le discours pour aboutir au 
changement souhaité, et ont constaté que ce qui avait été considéré jusqu'alors comme «non-rationnel» et «immoral», pouvait en réalité être conçu comme un comportement logique et stratégique qui se justifie par une situation d'inégalité. Cette constatation a contribué à une vision plus globale de la rhétorique, dont la théorie s'est intéressée aux conditions au sein desquelles les critères éthiques « traditionnels » sont valables. Elle a permis de se confronter aux limites du jugement éthique de l'orateur "dissident», celui qui viole toutes les règles de la rhétorique "propre». Cette démarche n'a pas seulement ouvert la voie à une série de questions concernant la validité de l'idéal démocratique du pluralisme d'idées et d'orateurs. Elle a également impliqué, pour les chercheurs, le souci d'exposer les inégalités à l'intérieur du système social d'opportunités comme dans l'arène publique. Dans cette perspective, les premiers travaux en rhétorique des mouvements sociaux ont bien insisté sur la fonction émancipatrice de la parole dissidente, et ont exprimé la volonté d'assurer l'ouverture à tous des "canaux de communication rationnelle ». L'étude des rapports de force qui existent dans l'espace discursif devient une condition préalable de l'analyse des présupposés, des tactiques et des objectifs de la rhétorique protestataire. Elle suppose aussi un certain type d'engagement social : si l'on croit en la pertinence de la communication rhétorique, il est impératif de lutter pour la démocratisation de l'espace rhétorique; et si l'on contribue à la formation d'orateurs habilités, il est indispensable de contribuer à la formation d'un espace rhétorique pluraliste et ouvert.

La nouvelle génération a élargi la perspective en s'attaquant de plus près aux enjeux idéologiques des mouvements étudiés. Depuis les années 1980, les chercheurs se demandent jusqu'à quel point leur propre travail contribue à la vie du mouvement, d'abord en le proposant à l'attention de la communauté scientifique, puis en cernant et interprétant sa signification et sa valeur symbolique à travers une analyse rhétorique. Admettant l'existence de rapports dialectiques entre le mouvement et son étude, c'està-dire entre la praxis et la théorie, ils commencent à s'interroger sur leur rôle à l'égard de l'évolution et du fonctionnement du mouvement. À ce stade, la "passion pour l'action » peut devenir plus que de l'empathie envers les militants et leur cause : c'est un type de participation. Plus récemment, un courant plus progressiste dans les études rhétoriques, en accord avec un vaste mouvement visant à repenser les rapports entre l'académie et l'activisme (Hale 2008), postule qu'aucune production scientifique n'est "objective ", et s'autorise en conséquence à réorganiser ses principes de travail en fonction de la subjectivité affichée, explicitée et argumentée du chercheur. En conséquence, le chercheur n'a pas seulement un "rôle générateur " à l'égard du mouvement qu'il étudie, il a également une obligation sociale par rapport à lui. Il est ouvertement appelé à mobiliser ses compétences en vue d'une transformation sociale et culturelle à travers son engagement, en tant que «activiste-académique » dans les mouvements sociaux.

N'ayant pas l'ambition et la prétention de critiquer ici l'une de ces deux approches ni d'en évaluer la pertinence, nous nous contenterons de deux remarques. La première concerne la spécificité de la posture des chercheurs en rhétorique des mouvements sociaux. Le survol historique sur le débat autour de leur responsabilité scientifique et sociale montre que leurs réflexions théoriques préfiguraient en grande partie certains courants scientifiques. D'abord, ceux qui animent l'étude de l'action collective depuis les années 1970-80, et surtout les approches qui conçoivent l'individu et le groupe en lutte comme des êtres rationnels et logiques; puis le tournant discursif dans la sociologie des mouvements sociaux, qui, depuis les années 1990, place les dynamiques 
persuasives et la construction du sens au centre de l'analyse sociologique de l'action collective. Ensuite, la nécessité d'examiner la situation rhétorique des mouvements sociaux a également entraîné le besoin de critiquer non seulement le caractère inégalitaire de l'espace public, mais aussi les intérêts au fondement de la production et de la diffusion du discours. Il s'avère qu'en s'intéressant à la rhétorique des mouvements sociaux, les chercheurs ont bien rendu compte du fait que celle-ci est conçue et produite avant tout face à un régime discursif plus large, et qu'aucune analyse rhétorique de protestation ne peut être menée à bien sans tenir compte du discours institutionnel du Pouvoir. Dans cette perspective, les réflexions théoriques des rhétoriciens préfiguraient les enjeux théoriques de mouvements critiques plus tardifs en linguistique et en sociolinguistique, comme la Critical Linguistic (CL) et le Critical Discourse Analysis (CDA). Il ne s'agit pas ici de revendiquer un droit de propriété, mais d'affirmer, historiquement, l'hypothèse avancée par Sharon McKenzie Stevens et Patricia Malesh (2009) selon laquelle les chercheurs en rhétorique sont bien placés pour mener une "méta-enquête" (meta-inquiry, 11) sur les dynamiques linguistiques, herméneutiques et persuasives qui animent les processus du changement social. Somme toute, c'est dans l'esprit de l'article de Griffin de 1952, que les chercheurs en rhétorique des mouvements sociaux ont organisé leurs premiers travaux autour de deux hypothèses très novatrices dans les années 1960 : que l'orateur dissident est un être rationnel qui agit de manière stratégique, et que, loin d'être pluraliste, l'arène publique est largement subordonnée aux intérêts de l'élite politique et économique.

Notre deuxième remarque concerne les limites de la responsabilité sociale. Dans la première génération, le souci dominant des rhétoriciens a été de mettre à jour leur théorie face à une réalité dynamique qui a exposé les défauts du système politique. L'ambition, implicite, était d'éclairer, à travers l'analyse rhétorique, ces défauts - non pas pour détruire l'idéal rhétorique, mais plutôt pour reconstruire un espace public pluraliste où l'idéal rhétorique pourrait être réalisé de manière équitable et ouverte. En tant que rhétoriciens, la responsabilité sociale des chercheurs était non seulement de "dicter" les règles de la "bonne" rhétorique, mais aussi de contribuer à la constitution d'un espace où ces règles deviennent réellement efficaces.

La génération la plus récente prend pour objectif principal de promouvoir le changement social à travers des recherches et un enseignement explicitement orientés. Cet idéal mobilise le travail universitaire au service d'une mission globale de «transformation sociale et culturelle » de la société ; mais en réalité, derrière cet idéal, on relève souvent un ordre du jour idéologique et politique très marqué. Cette tendance n'est pas ignorée des chercheurs. Citant Nelson Pichardo (1997 : 413), qui a constaté que les chercheurs travaillant sur de l'action collective «ont souvent marginalisé les mouvements sociaux qui ne proviennent pas de la gauche », l'essai de clôture d'Active Voices, rédigé par William DeGenero, regrette l'absence des mouvements de la droite américaine dans cet ouvrage collectif. Au-delà de ce livre, cette constatation reste toujours valable, mais elle est incomplète ${ }^{14}$. "Agentivité ", "Critique ", "Situation rhétorique »: ces termes disparaissent lorsqu'on étudie la rhétorique des mouvements de la droite américaine, où les mêmes formes de discours menaces, appels aux émotions, diabolisation de l'adversaire, coercition - servent très souvent d'exemple d'une rhétorique " populiste » (voir, à titre d'exemple, Enck-Wazner 2011) ou " démagogique » (voir Hogan \& Tell 2006: 483). Nous n'avons pas l'intention de porter un jugement sur ce phénomène, qui demande d'ailleurs à être encore empiriquement validé. Mais si cette observation était confirmée, le domaine de la 
rhétorique des mouvements sociaux serait à nouveau soumis à un régime de jugement éthique, où les présupposés par rapport à la persuasion, et surtout les distinctions entre « rhétorique » et "propagande » et entre discours « raisonnés » ou « démagogiques", seraient largement basés sur des critères politiques et reflèteraient la division gauche / droite.

De plus, si l'on admet le rôle "générateur " de l'analyse rhétorique des mouvements sociaux, le fait que le choix du chercheur joue un rôle dans la diffusion du mouvement analysé et influence la hiérarchisation des problèmes sociaux dans la société, et si on reconnaît la "subjectivité explicitée» du chercheur ${ }^{15}$, il convient de se poser les questions suivantes : à partir de quel moment l'engagement du chercheur dans sa mission de changement social en vient-il à subordonner son travail à un ordre du jour politique? Quel espace d'expression est offert aux étudiants, collègues, et partenaires qui ne partagent pas telle ou telle perspective sur la réalité sociale? Surtout, à quel critère se soumettent les «activistes académiques » dans leur travail universitaire : le critère académique ou le critère militant?

Soucieuses de ce que la classe ne se transforme pas en un lieu de "conversion", les éditrices d'Active Voices affirment bien que «si [nos étudiants] quittent nos classes en imitant notre propre système de compréhension au lieu de découvrir et développer le leur comme un moyen de s'engager dans une transformation structurale, nous avons échoué » (2009: 16).

Cette affirmation mérite, à notre avis, d'être élaborée. Dans la mesure où Active Voices et Activism and Rhetoric se présentent comme des textes programmatiques avançant et démontrant les modalités de militantisme et d'engagement des rhétoriciens, il est également pertinent qu'ils s'interrogent sur les limites de l'engagement, et surtout sur les conséquences de la "subjectivité » ouverte, à savoir la politisation explicite des chercheurs-enseignants. Cette question dépasse les limites de la réflexion pédagogique, et ne concerne pas uniquement la conscience politique des étudiants. Dans la mesure où la rhétorique des mouvements sociaux, comme la sociologie de l'action collective, "marginalise » désormais les mouvements qui ne correspondent pas aux croyances politiques des chercheurs et les stigmatise comme "démagogiques", les rhétoriciens des mouvements sociaux doivent, nous semble-t-il, veiller à ce que leur propre champ de discours soit le plus pluraliste et ouvert que possible.

Mais la dichotomie gauche / droite et les « risques » de la politisation (à gauche) de la rhétorique des mouvements sociaux, ne doivent pas désorienter le débat. Fondamentalement, l'appel de la nouvelle génération reste très pertinent pour quiconque se réclame d'une tendance politique et idéologique qui reconnait la valeur d'une agentivité rhétorique. La mission des chercheurs consiste à conférer à tous, étudiants, collègues, citoyens, une connaissance théorique leur permettant de rendre compte de leur propre agentivité rhétorique, et de les guider à travers leur transformation en agents rhétoriques autonomes.

Dans son article d'Active Voices, Sharon McKenzie Stevens reprend la notion de «situation rhétorique » pour formuler ainsi la question de la responsabilité : c'est au « rhétor » de " choisir entre reproduction sociale et changement » et de décider s'il est pertinent ou non d'" accepter la situation rhétorique comme telle, y compris les rapports sociaux qui la construisent» (51). Ceux qui agissent en faveur d'un changement social font face à un dilemme cardinal : adapter leur rhétorique au régime de savoir et au décorum discursif existants, ou bien aller plus loin et tenter de modifier 
ceux-ci. Le chercheur et l'enseignant sont plutôt chargés de fournir à la communauté scientifique et à la société les moyens nécessaires pour faire un choix informé et raisonné - et éthique - par rapport à ce dilemme. Ainsi, si la rhétorique des mouvements sociaux a une responsabilité sociale précise, elle se trouve quelque part entre les deux approches décrites plus haut, et nous la résumons ainsi : la formation de l'agentivité rhétorique autonome dans une situation rhétorique dont on est pleinement capable de critiquer les enjeux politiques et sociaux.

\section{BIBLIOGRAPHIE}

Bosmajian, Haig A. [Éd.] (1971). Dissent : Symbolic Behavior and Rhetorical Strategies (Boston : Allyn and Bacon)

Bowers, John Waite \& Donovan J. Ochs (1971). The Rhetoric of Agitation and Control (Reading, MA : Addison-Wesley)

Burgess, Parke G. (1968). « The Rhetoric of Black Power : A Moral Demand ? », Quarterly Journal of Speech $54: 2,122-133$

Burgess, Parke G. (1970). « The Rhetoric of Moral Conflict : Two Critical Dimensions », Quarterly Journal of Speech $56: 2,120-130$

Burgess, Parke G. (1973) «Crisis Rhetoric : Coercion vs. Force », Quarterly Journal of Speech $59: 1$, 61-73

Calhoun, Craig \& Michel Wieviorka (2013). « Manifeste pour les sciences sociales », Socio 1, 3-38 [en ligne : http://socio.hypotheses.org/147]

Campbell, Karlyn Kohrs (1971). « The Rhetoric of Radical Black Nationalism : A Case Study in SelfConscious Criticism », Central States Speech Journal 22 : 3, 151-160

Campbell, Karlyn Kohrs (1973), « The Rhetoric of Womens' Liberation : An Oxymoron », Quarterly Journal of Speech $59: 1,74-86$

Cathcart, Robert. S. (1978). « Movements : Confrontation as Rhetorical Form », Southern Speech Communication Journal, $43: 3,233-247$

Chazel, François (2009). « Mobilisation des ressources », in Olivier Fillieule, Lilian Mathieu \& Cécile Péchu (Éds), Dictionnaire des mouvements sociaux (Paris : Presses de Sciences-Po), pp. 364-370

Cloud, Dana (2011a). We are The Union: Democratic Unionism and Dissent at Boeing (Urbana, IL: University of Illinois Press)

Cloud, Dana (2011b). « The Only Conceivable Thing To Do : Reflections on Academics and Activism », in Seth Kahn \& JongHwa Lee (Éds), Activism and Rhetoric : Theories and Contexts for Political Engagement (New-York : Routledge), pp. 11-24

Cox, Robert \& Christina R. Foust (2009), « Social Movement Rhetoric », in Andrea A. Lunsford, Kirt H. Wilson \& Rosa A. Eberly (Éds.), The SAGE Handbook of Rhetorical Studies (Los Angeles : Sage Publications), pp. 605-622 
DeGenero, William (2009). « Politics, Class, and Social Movement People : Continuing the Conversation ", inSharon McKenzie Stevens \& Patricia Malesh (Éds), Active Voices : Composing a Rhetoric for Social Movements (Albany, NY : SUNY), pp. 199-2011

DeLuca, Kevin Michael (1999a). Image Politics. The New Rhetoric of Environmental Activism (New-York et London : Guilford Press)

DeLuca, Kevin Micheal (1999b). « Unruly Arguments : The Body Rhetoric of Earth First!, ACT UP, and Queer Nation ", Argumentation and Advocacy $36: 1,9-21$

Denton, Robert E. (1980). « The Rhetorical Functions of Slogans : Classifications and Characteristics ", Communication Quarterly 28: 2, 10-18

Doumani, Beshara (2006). « Between Coercion and Privatization : Academic Freedom in the Twenty-First Century ", in Beshara Doumani (Éd.), Academic Freedom After September 11 (NewYork : Zone Books), pp. 11-57

Doury, Marianne (2004) « La position du chercheur en argumentation », SEMEN 17, 149-163 [en ligne : http://semen.revues.org/2345]

Enck-Wanzer, Darrel (2006). « Trashing the System : Social Movement, Intersectional Rhetoric, and Collective Agency in the Young Lords Organization's Garbage Offensive », Quarterly Journal of Speech $92: 2,174-201$

Enck-Wazner, Darrel (2011). « Barack Obama, the Tea Party, and the Threat of Race : On Racial Neoliberalism and Born Again Racism », Communication, Culture and Critique $4: 1,23-30$

Foust, Christina R. (2010). Transgression as a Mode of Resistance: Rethinking Social Movement in an Era of Corporate Globalization (Lanham, MD: Lexington Books)

Gamson, William A. (1975). The Strategy of Social Protest (Hemewood, IL : Dorsey Press)

Golden, James L. \& Richard D. Rieke (1971). The Rhetoric of Black Americans (Columbus, Oh: C. E. Merrill)

Gregg, Richard B. (1971). « The Ego-Function in the Rhetoric of Protest », Philosophy and Rhetoric $4: 2,71-91$

Griffin, Leland M. (1952). « The Rhetoric of Historical Movement », Quarterly Journal of Speech 38 : 2, 184-188

Griffin, Leland M. (1964). « The Rhetorical Structure of the "New Left" Movement : Part I », Quarterly Journal of Speech $50: 2,113-135$

Haiman, Franklyn S. Haiman (1967). “'The Rhetoric of the Streets' : Some Legal and Ethical Considerations ", Quarterly Journal of Speech $53: 2,99-114$.

Hale, Charles R. [Éd.] (2008). Engaging Contradictions : Theory, Politics, and Methods of Activist Scholarship (Berkeley : University of California Press)

Hauser, Gerard A. \& erin daina mcclellan (2009). « Vernacular Rhetoric and Social Movements : Performances of Resistance in the Rhetoric of the Everyday », in Sharon McKenzie Stevens \& Patricia Malesh (Éds), Active Voices: Composing a Rhetoric for Social Movements (Albany, NY: SUNY), pp. 23-46

Hogan, J. Michael \& Dave Tell (2006). « Demagoguery and Democratic Deliberation : The Search for Rules of Discursive Engagement », Rhetoric \& Public Affairs $9: 3,479-487$

Jensen, Richard J. (2001). « Evolving Protest Rhetoric : From the 1960s to the 1990s », Rhetoric Review $20: 1 / 2,28-32$ 
Jensen, Richard J. (2006). « Analyzing Social Movement Rhetoric », Rhetoric Review 25 : 4, 372-375

Kampbell, Karlyn Kohrs (1973). «The Rhetoric of Women's Liberation : An Oxymoron », Quarterly Journal of Speech $59: 1,74-86$

Knupp, Ralph E. 1981. «A Time for Every Purpose under Heaven : Rhetorical Dimensions of Protest Music ", Southern Speech Communication Journal $46: 4,377-389$

Lake, Randall A. (1983). « Enacting Red Power : The Conssumatory Function in Native American Protest Rhetoric », Quarterly Journal of Speech $69: 2,127-142$

Lowi, Theodore J. (1971). The Politics of Disorder (New York : Basic Books)

Maingueneau, Dominique. 2012. «Que cherchent les analystes du discours ? », Argumentation et Analyse du Discours 9 [en ligne : http://aad.revues.org/1354]

McGee, Michael Calvin (1980). «'Social Movements' : Phenomenon of Meaning ? ", Central States Speech Journal $31: 4,233-244$

McKenzie Stevens, Sharon (2009). « Dreaming to Change Our Situation : Reconfiguring the Exigence for Student Writing ", in Sharon McKenzie Stevens \& Patricia Malesh (Éds), Active Voices : Composing a Rhetoric for Social Movements (Albany, NY : SUNY), pp. 47-65

McKenzie Stevens, Sharon \& Patricia Malesh (2009). «Introduction : Active Voices ", in Sharon McKenzie Stevens \& Patricia Malesh (Éds), Active Voices : Composing a Rhetoric for Social Movements (Albany, NY : SUNY), pp. 1-20

McKenzie Stevens, Sharon \& Patricia Malesh [Éds] (2009), Active Voices : Composing a Rhetoric for Social Movements (Albany, NY : SUNY)

Mills, C. Wright (1956). The Power Elite (New York : Oxford University Press)

Morris, Charles E. \& Stephen H. Browne [Éds.] (2001). Readings on The Rhetoric of Social Protest (State College, PA : Strata)

Pichardo, Nelson A. 1997. « New Social Movements : A Critical Review », Annual Review of Sociology 23, 411-430.

Railsback, Celeste Condit. (1984). «The Contemporary American Abortion Controversy : Stages in the Argument ", Quarterly Journal of Speech $70: 4,410-424$

Rothwell, J. Dan (1971). «Verbal Obscenity : Time for Second thoughts », Western Speech 35 : 4 , 231-242

Scott, Robert L. (1967). « On Viewing Rhetoric as Epistemic », Central States Speech Journal 18 : 1 , $9-17$

Scott, Robert L. \& Donald K. Smith (1969). « The Rhetoric of Confrontation », Quarterly Journal of Speech $55: 1,1-8$

Sillars, Malcolm O. (1976). «Persistent Problems in Rhetorical Criticism », Jane Blankenship \& Hermann G. Stelzner (Éds), Rhetoric and Communication : Studies in the University of Illinois Tradition (Urbana, IL : University of Illinois Press), pp. 69-88

Sillars, Malcolm O. (1980). « Defining movements rhetorically : Casting the widest net », Southern Speech Communication Journal $46: 1,17-32$

Simons, Herbert W. (1971). « Requirements, Problems, and Strategies : A theory of persuasion for social movements », Quarterly Journal of Speech $56: 1,1-11$ 
Stewart, Charles J. (1980). « A Functinal Approach to the Rhetoric of Social Movements ", Central States Speech Journal $31:$, 298-305

Stewart, Charles J., Craig Allen Smith \& Robert E. Denton (1984). Persuasion and Social Movements (Prospect Heights, IL : Waveland Press)

Zarefsky, David (1980). « A Sceptical View of Movement Studies », Central States Speech Journal 31 : 4, 245-254.

Zarefsky, David (2011). « Introduction : Michael Leff et la tradition des études rhétoriques aux Etats-Unis », Argumentation et Analyse du Discours 6 [en ligne : http://aad.revues.org/1082]

\section{NOTES}

1. Précisons qu'il existe des travaux sur le discours de protestation en France et en Europe qui reflètent des problématiques et des perspectives similaires, mais que cet article traite spécifiquement du cas américain, et prend pour objectif la description et l'analyse du débat tel qu'il s'exprime chez les chercheurs affiliés au domaine de la "rhétorique des mouvements sociaux » aux États-Unis.

2. Contrairement à la France, aux États-Unis la rhétorique est depuis longtemps un domaine de recherche et d'enseignement autonome et reconnu, dont les spécialistes se situent pour la plupart dans les départements de communication ou d'anglais des universités. Sur l'évolution du domaine et le tournant critique des années 1960, on lira l'article de David Zarefsky (2011), dans le numéro 6 de la revue Argumentation et Analyse du Discours, consacré à l'analyse rhétorique aux États-Unis.

3. Toutes les traductions de l'anglais sont de nous.

4. Sur l'évolution du domaine on consultera Cox et Foust 2009.

5. Cette aspiration à un modèle unifié a fait l'objet d'un séminaire de la Speech Communication Association en 1979, dont les interventions ont été publiées dans un numéro spécial de 1980 ( $\mathrm{n}^{\circ}$ 31, vol. 4) du Central States Speech Journal (devenu Communication Studies en 1988).

6. Voir, à titre d'illustration, le témoignage de Richard J. Jensen (2001).

7. Voir, à titre d'illustrations, les travaux et les documents réunis par Bosmajian 1971.

8. L'idée selon laquelle la critique rhétorique doit traiter de la manière dont le message rhétorique permet de "créer la vérité » (creating truth) et non pas seulement de «construire l'efficacité " (give effectiveness) d'une vérité préalablement donnée, est longuement développée dans un précédent article de Robert Scott (1967), qui introduit la notion de «Rhétorique épistémique » (Epistemic Rhetoric).

9. C'est à la même période que politologues et sociologues commencent à dresser leur propre bilan sur la « structure des opportunités ». À l'instar de Mills (1956) et Lowi (1971), Gamson (1975) affirme dans son célèbre The Strategy of Social Protest, que la protestation des années 1960 a été mal vue par les Américains, qui, persuadés de la nature ouverte et pluraliste de leur société, ont perçu l'agitation sociale comme une subversion du système démocratique. Sur cet écart entre l'idéal démocratique et la réalité, on lira le chapitre 9 de l'ouvrage de Gamson, «The Limits of Pluralism ».

10. L'auteur reprend explicitement ici le vocabulaire de la Nouvelle Rhétorique de Perelman et Olbrechts-Tyteca.

11. Sur la notion de pluralisme culturel en rhétorique on lira Campbell 1971 et Golden et Rieke (1971). 
12. L'auteur évoque ici l'opposition entre l'approche stratégique et l'approche éthique (ou moraliste) dans la critique rhétorique. Pour une discussion relative à la période, voir Sillars (1976: 77-81).

13. Dans leur «Manifeste pour les sciences sociales ", Craig Calhoun et Michel Wieviorka (2013) contestent, entre autres, cette fragmentation du savoir imposée par la structure institutionnelle des systèmes universitaires.

14. Dominique Maingueneau (2012) constate un phénomène similaire dans l'analyse du discours en France, et affirme qu'« à ses débuts l'analyse du discours française a privilégié les corpus politiques et parmi eux presque exclusivement ceux empruntés à la gauche; il a fallu attendre les années 1980 pour que les corpus de droite soient pris en compte, et encore s'agit-il essentiellement de l'extrême droite » (57).

15. Dana Cloud se définit, dans son article de 2011, comme « socialiste de longue date » (longtime socialist) (15).

\section{RÉSUMÉS}

Depuis les années 1960 il existe aux États-Unis un domaine de recherche distinct au sein des études rhétoriques qui explore la « rhétorique des mouvements sociaux ». Les chercheurs affiliés à ce courant mènent, dès le départ, un débat riche et programmatique qui traite de problématiques métathéoriques liées à la responsabilité scientifique du chercheur et à la fonction sociale de la recherche dans le contexte de la protestation et de l'action collective. Cet article explore l'évolution de ce débat, dont les participants ont dans un premier temps contesté la pertinence de l'ethos scientifique de la théorie rhétorique dans l'époque contemporaine, et ont aspiré à redéfinir la responsabilité du chercheur par rapport au fonctionnement de l'espace discursif. Par la suite, les chercheurs en rhétorique des mouvements sociaux ont examiné les rapports dialectiques entre l'analyse rhétorique et les mouvements étudiés, c'est-à-dire entre la théorie et la pratique, et en conséquence ont aspiré à formuler l'engagement des spécialistes en rhétorique des mouvements sociaux à la fois comme chercheurs et enseignants. L'article décrit ce débat et traite de sa contribution, d'une part à l'évolution de la théorie rhétorique, et d'autre part à la réflexion sur l'engagement scientifique et social du chercheur en tout ce qui concerne les processus et les modalités du changement social.

Since the 1960s, there has been an active and distinct research field within rhetorical studies in the United States, which explores the "rhetoric of social movements". Researchers affiliated with this stream have staged from the outset a rich and structured debate dealing with metatheoretical issues related to the scientific responsibility of the researcher and the social role of the research in the context of protest and collective action. This article explores the evolution of this debate, in which the participants have initially questioned the relevance of the scientific ethos of contemporary rhetorical theory, and aspired to redefine the responsibility of the researcher with regard to the social dynamics in the public sphere. Subsequently, researchers in the rhetoric of social movements discussed the dialectical relations between rhetorical analysis and social movements, i.e. between theory and practice, and therefore aspired to define the social commitment of specialists in the rhetoric of social movements both as researchers and teachers. The article describes this debate and discusses its contribution to the evolution of 
rhetorical theory on the one hand, and to the ongoing thinking about the scientific and social commitment of the researcher vis-a-vis processes and practice of social change on the other.

INDEX

Mots-clés : agentivité (rhétorique), engagement scientifique, fonction sociale de la recherche, rhétorique des mouvements sociaux

Keywords : agency (rhetorical), scientific commitment, social function of research, social movement rhetoric

\section{AUTEUR}

EITHAN ORKIBI

Université d'Ariel, ADARR 